\title{
Estudo cinético da degradação térmica e catalítica de polipropileno sob catalisadores mesoporosos tipo AlMCM-48
}

Kinetic study of thermal and catalytic degradation of polypropylene over mesoporous catalysts type AlMCM-48

\author{
T. G. dos Santos ${ }^{1,2 *}$; A. O. S. Silva ${ }^{2}$; A. S. Araujo ${ }^{3}$; M. J. B. Souza ${ }^{4}$ \\ ${ }^{1}$ Grupo de Catálise e Reatividade Química-GCaR, Universidade Federal de Alagoas, 57072-970, Maceió-AL, Brasil \\ ${ }^{2}$ Laboratório de Síntese de Catalisadores-LSCat, Universidade Federal de Alagoas,57072-970, Maceió-AL, Brasil \\ ${ }^{3}$ Laboratório de Catálise e Petroquímica-LCP, Universidade Federal de Rio Grande do Norte, 49100-970, Natal-RN, \\ Brasil \\ ${ }^{4}$ Laboratório de Catálise-LabCat, Universidade Federal de Sergipe, 49100-000, São Cristóvão-SE, Brasil
}

*tiago.santos@iqb.ufal.br

(Recebido em 29 de abril de 2021; aceito em 20 de setembro de 2021)

O lixo plástico sofreu um aumento drástico e se tornou um dos maiores problemas ambientais do século XXI. A degradação térmica e catalítica de plásticos é uma das soluções viáveis para esse problema, pois pode conduzir tanto a benefícios de proteção ambiental, quanto para processos economicamente vantajosos em termos de gastos de energia. Neste trabalho, é apresentado um estudo termogravimétrico da conversão do polipropileno (PP) na presença de catalisadores mesoporosos AlMCM-48 ( $\mathrm{Si} / \mathrm{Al}=50$ e 100). O desempenho catalítico mostrou que a reação de degradação do PP ocorreu em etapas de reações complexas nas taxas de aquecimento 5, 10 e $20{ }^{\circ} \mathrm{C} \mathrm{min}^{-1}$. Além disso, o volume dos poros dos catalisadores nas diferentes razões molares $\mathrm{Si} / \mathrm{Al}$ influenciou o processo de degradação em condições não isotérmicas e desempenhou um papel importante na diminuição da temperatura de degradação do PP. A energia de ativação aparente $\left(E_{a}\right)$ foi determinada pelo método de isoconversão de Vyazovkin. Os parâmetros cinéticos obtidos revelaram uma redução na $E_{a}$ evidenciando uma média de 89,66 e 59 kJ mol $^{-1}$ para a degradação do PP puro, PP + AlMCM-48(50) e PP + AlMCM-48(100), respectivamente. Como mostrado pelos valores médios, ficou evidenciado que a maior eficiência foi do AlMCM-48(100) que teve uma diminuição da energia de ativação de cerca de $30 \mathrm{~kJ} \mathrm{~mol}^{-1}$ em relação a energia de ativação do PP puro.

Palavras-chave: AlMCM-48, polipropileno, cinética de isoconversão.

The plastic waste has suffered a dramatically increase and has become one of the biggest environmental problems of the 21 st century. Thermal and catalytic degradation of plastics is one of the viable solutions to this problem since it can lead to both environmental protection benefits and economically advantageous processes in terms of energy expenditure. In this article a thermogravimetric study of the conversion of polypropylene (PP) in the presence of AlMCM-48 mesoporous catalysts $(\mathrm{Si} / \mathrm{Al}=50$ and $100)$ is presented. The catalytic performance showed that the PP degradation reaction occurs in complex reaction steps in the heating rates 5,10 and $20{ }^{\circ} \mathrm{C} \mathrm{min}^{-1}$. Moreover, the pore volume of the catalysts in the different molar ratios $\mathrm{Si} / \mathrm{Al}$ influenced the degradation process under non-isothermal conditions and played an important role in decreasing the degradation temperature of the PP. The apparent activation energy $\left(\mathrm{E}_{\mathrm{a}}\right)$ was determined by the Vyazovkin isoconversion method. The kinetic parameters obtained revealed a reduction in $E_{a}$, showing an average of 89,66 and $59 \mathrm{~kJ} \mathrm{~mol}^{-1}$ for the degradation of pure PP, PP + AlMCM-48(50) and PP + AlMCM-48(100), respectively. As shown by the average values, it was evidenced that the greatest efficiency was of the AlMCM-48(100) that had a decrease of the activation energy of about $30 \mathrm{~kJ} \mathrm{~mol}^{-1}$ when compared with the pure PP.

Keywords: AlMCM-48, polypropylene, isoconversion kinetics.

\section{INTRODUÇÃO}

O desenvolvimento da indústria petroquímica resulta não apenas em uma gama de produtos úteis para as atividades humanas, mas também no aumento de resíduos para o meio ambiente, pois os produtos usados na maioria das vezes são descartados de forma inapropriada após o consumo. Um exemplo é o polipropileno (PP), um dos plásticos mais comuns, com inúmeras aplicações industriais que vão desde embalagens a eletrodomésticos, roupas e veículos. Essa 
promoção decorre de suas propriedades versáteis e preço relativamente baixo [1, 2]. Este material é o segundo plástico mais produzido no mundo, atrás do polietileno, com 55 milhões de toneladas consumidas globalmente em 2013 e a expectativa é que a demanda por esse tipo de plástico cresça em uma média de 4,9\% ao ano até 2026 [3]. No geral, estima-se que desde 2020 mais de 400 milhões de toneladas de resíduos plásticos foram gerados anualmente e a produção de plásticos deve dobrar até 2035, atingindo 800 milhões de toneladas e quase 1600 milhões de toneladas até $2050[4,5]$. Com relação ao seu descarte, 6,3 bilhões de toneladas métricas da produção global de plástico de 1950 a 2018 terminaram como resíduos, destes cerca de 4,5 bilhões de toneladas acabaram em aterros sanitários [6], que tem como consequência, a infertilidade do solo uma vez que são necessários mais de 500 anos para a degradação completa [7] e liberação de toxinas [8]. Estes dados confirmam que a poluição por plásticos é um dos problemas ambientais mais urgentes que a humanidade enfrenta neste século.

Diante deste cenário preocupante, estudos destacam o potencial da degradação termocatalítica de resíduos plásticos [9-12]. O processo catalítico permite que a degradação do plástico seja realizada em temperaturas mais baixas [12]. Além disso, a conversão catalítica desses resíduos plásticos em produtos químicos valiosos pode caracterizar uma solução eficiente para o problema da poluição e uma boa alternativa à utilização dos combustíveis fósseis [9]. Os catalisadores mais comumente usados na degradação termo-catalítica de plásticos são zeólitas [13-15] e sílica alumina [16, 17]. Vyas et al. (2016) [18] analisaram o craqueamento de PP usando diferentes catalisadores que incluíam ZSM-5 e uma zeólita de ocorrência natural em um reator fabricado com sistema de leito fluidizado com uma disposição para medir a temperatura de $100-800{ }^{\circ} \mathrm{C}$. Os experimentos de craqueamento foram executados sob diferentes proporções de PP:catalisador (de 1:1 até 5:1). Os autores verificaram que a proporção mais eficaz de PP:catalisador foi de 3:1 na temperatura variando de $420-510{ }^{\circ} \mathrm{C}$ com a ZSM-5, que apresentou maior conversão de produto residual de PP na forma líquida. As zeólitas apresentam propriedades que as tornam excelentes catalisadores para a degradação termo-catalítica de plásticos devido à sua elevada acidez, o que é uma característica desejada para a cisão da ligação C-C. No entanto, o tamanho dos poros desses catalisadores é limitado a um valor máximo de cerca de $1,0 \mathrm{~nm}$, o que impede a entrada de subtratos volumosos aos sítios ativos [19]. Neste sentido, desde 1992, o desenvolvimento de catalisadores na faixa de mesoporosidade (diâmetro de poro entre 2,0 e 50,0 nm) tem sido explorado, a exemplo da família M41S, que é constituída pelas peneiras moleculares MCM-41, MCM-48 e MCM-50 [20].

A peneira molecular MCM-48 é o segundo material mais estudado da família M41S e apresenta uma cela unitária de simetria cúbica, com um arranjo de canais tridimensionais, o qual consiste em duas redes contínuas interpenetrantes de canais quirais [21]. Estes pares de canais porosos não sobreponíveis são conhecidos por estarem desagregados por uma parede inorgânica que segue exatamente um giróide de superfície mínima periódica infinita [22]. O arranjo de canais porosos da MCM-48 pode ser entendido também como sendo um conjunto de canais intergeminados que não se comunicam. Esta rede tridimensional (3D) altamente porosa pode fornecer uma maior acessibilidade para moléculas volumosas aos sítios ativos, propiciando a difusão ao longo dos poros sem bloqueá-los. Isso é o que tem incentivado o estudo e desenvolvimento de novas pesquisas no que tange à produção de catalisadores com alto grau de ordenação por meio da incorporação de espécies metálicas na estrutura da MCM-48 para a introdução de sítios ativos e, o que melhora, consequentemente, o desempenho catalítico [23, 24]. No entanto, a literatura reporta que a presença de espécies metálicas pode comprometer significativamente a estrutura de materiais à base de sílica causando desordem na rede ou colapso parcial da estrutura [25].

Esforços consideráveis têm sido desprendidos no desenvolvimento de AlMCM-48 com várias fontes de alumínio (pseudoboemita [12], sulfato de $\mathrm{Al}$ [23, 26-30], isopropóxido de $\mathrm{Al}$ [26, 27, 31, 32], aluminato de sódio [23, 27, 29, 33, 34], hidróxido de Al [29, 35, 36], tertbutóxido de $\mathrm{Al}$ [26], tri-sec-butóxido de $\mathrm{Al}$ [37], nitrato de $\mathrm{Al}$ e triisopropilato de $\mathrm{Al}$ [29]). As espécies ativas geradas podem ser aplicadas em diversos processos como isopropilação de naftaleno [35], isopropilação de fenol com acetato de isopropila [36], síntese de $\varepsilon$-caprolactama a partir da oxima de ciclohexanona [34], pirólise de biomassa [38], craqueamento de 
hexadecano e dodecano [23]. Além disso, devido às suas propriedades de acidez e tamanho de poro, suas aplicações na degradação de plásticos, como polietileno linear de baixa densidade [39], polipropileno [40], polietileno de alta densidade [12] e polietileno de alta densidade como co-alimentação da matéria-prima na pirólise catalítica da biomassa [41] são relevantes.

O conhecimento sobre análise térmica exerce um fator importante na determinação das condições de processamento e reciclagem de materiais plásticos [42, 43]. Neste sentido, a análise termogravimétrica (TG) é uma das técnicas comumente usadas para investigar tais processos, no que tange às reações de degradação térmica e catalítica [12]. Desta forma, utilizando-se de procedimentos experimentais adequados, informações sobre a cinética de degradação via TG podem ser obtidas $[9,10,44]$, bem como uma previsão da energia de ativação aparente quantificada a partir da aplicação de modelos matemáticos (model-fitting e model-free kinetics) [45, 46]. É importante notar que a energia de ativação derivada dos métodos conhecidos como model-free kinetics é mais confiável uma vez que não há necessidade de assumir quaisquer funções do mecanismo de reação para calcular os parâmetros cinéticos e investigar o processo de degradação de plásticos [45, 47].

Portanto, neste trabalho, a influência e o desempenho do AlMCM-48 na degradação térmica e catalítica do PP foram investigados em condições não isotérmicas. Pelo melhor do nosso conhecimento, raros são os estudos nesta área disponíveis na literatura com a finalidade de avaliar o efeito de catalisadores do tipo AlMCM-48 na degradação do PP em diferentes taxas de aquecimento $\left(5,10\right.$ e $\left.20{ }^{\circ} \mathrm{C} \mathrm{min}^{-1}\right)$. Além disso, a cinética de degradação foi estudada em detalhes empregando-se a abordagem de isoconversão de Vyazovkin, um método do model-free kinetics, que permitiu a coleta de dados cinéticos a partir das curvas TG pela regressão de $\ln \left(\beta / \mathrm{T}^{2}\right)$ em função de $1 / \mathrm{T}\left(\mathrm{K}^{-1}\right)$ para obtenção da energia de ativação aparente dos sistemas: PP puro e PP misturado fisicamente com as amostras de catalisadores, codificados como PP + AlMCM-48(50) e PP + AlMCM-48(100); onde, 50 e 100 representam a razão molar Si/Al.

\section{MATERIAL E MÉTODOS}

\subsection{Materiais}

Os materiais precursores e reagentes usados neste trabalho foram: tetraetil ortossilicato (TEOS, $\mathrm{C}_{8} \mathrm{H}_{20} \mathrm{O}_{4} \mathrm{Si}$, Aldrich, 99\%), brometo de cetiltrimetilamônio (CTMABr, $\mathrm{C}_{19} \mathrm{H}_{42} \mathrm{BrN}$, Vetec, 99\%), pseudoboemita (óxido-hidróxido de alumínio, $\mathrm{Al}_{2} \mathrm{O}_{3} \cdot \mathrm{H}_{2} \mathrm{O}$, Vista Chemical, $70 \%$ $\left.\mathrm{Al}_{2} \mathrm{O}_{3}\right)$, hidróxido de sódio $(\mathrm{NaOH}$, Vetec, $99 \%)$ sem etapa de pré-tratamento. A resina plástica de PP selecionada para este estudo foi adquirida de uma empresa produtora de polímeros de Maceió-AL, Brasil.

\subsection{Síntese hidrotérmica do AlMCM-48}

A estratégia de síntese foi relatada na literatura [12]. A composição do gel de síntese utilizada foi: 0,5950 CTMABr : 0,26 NaOH : 1 TEOS : $72 \mathrm{H}_{2} \mathrm{O}: \mathrm{y}_{2} \mathrm{Ol}_{3}$; onde, y=0,010 e 0,005 , correspondendo às razões molares Si/Al teóricas de 50 e 100, respectivamente. Sob condições hidrotermais, pH 12 e razão CTMABr/TEOS de 0,595, o gel de síntese foi transferido para autoclaves, aquecido em um forno à temperatura de $140{ }^{\circ} \mathrm{C}$ sob pressão autógena e condições estáticas por $72 \mathrm{~h}$. Após o tempo de envelhecimento, as autoclaves foram resfriadas até temperatura ambiente. Depois disso, o produto sólido foi separado do sobrenadante por filtração, lavagem com água destilada, seco a $100{ }^{\circ} \mathrm{C}$ por $6 \mathrm{~h}$ e calcinado a 450 ${ }^{\circ} \mathrm{C}$ por $2 \mathrm{~h}$ em ar com uma taxa de aquecimento de $10{ }^{\circ} \mathrm{C} \mathrm{min}^{-1}$. As amostras modificadas com $\mathrm{Al}$ foram denotadas como AlMCM-48(x) e x representa a razão molar Si/Al.

\subsection{Caracterização}

As amostras calcinadas foram analisadas por difratometria de raios $\mathrm{X}$ (DRX) em 
difratômetro Shimadzu, modelo XRD-6000, no intervalo de ângulo de Bragg de 20=1,5-10 . As medições de adsorção-dessorção de nitrogênio foram realizadas a $-196{ }^{\circ} \mathrm{C}(77,15 \mathrm{~K}) \mathrm{em}$ analisador Micromeritics, modelo ASAP-2020. As propriedades texturais foram estimadas usando-se a equação de Brunauer-Emmett-Teller (BET) e o método de Barrett-Joyner-Halenda $(\mathrm{BJH})$. As análises termogravimétricas (TG/DTG) foram realizadas usando-se analisador Shimadzu, modelo DTG-60H, com vazão de $\mathrm{N}_{2}$ de $40 \mathrm{~mL} \mathrm{~min}^{-1}$ e faixa de temperatura de 30$900{ }^{\circ} \mathrm{C}$ a uma taxa de aquecimento de $10{ }^{\circ} \mathrm{C} \mathrm{min}^{-1}$. A espectrometria de energia dispersiva de raios $\mathrm{X}(\mathrm{EDX})$ foi a técnica usada para se determinar a quantidade de $\mathrm{Al}_{2} \mathrm{O}_{3}$ das amostras por meio de um espectrômetro Shimadzu-EDX-7000/8000. Os espectros no infravermelho com transformada de Fourier (FTIR) foram registrados em equipamento Shimadzu IR Prestige 21 na faixa de número de onda de $4000-400 \mathrm{~cm}^{-1}$ e resolução de $4,0 \mathrm{~cm}^{-1}$. Imagens de microscopia eletrônica das amostras foram obtidas usando-se um microscópio de varredura TESCAN, modelo VEGA3, e um microscópio de transmissão FEI Tecnai G2 Spirit TWIN (120 kV). Detalhes dos resultados dessas caracterizações podem ser visualizados no trabalho de Dos Santos et al. (2021) [12].

\subsection{Degradação catalítica do PP via TG}

Anteriormente aos testes, o PP foi congelado com nitrogênio líquido para obtenção de materiais mais densos, moído e peneirado até uma granulometria superior a 35 mesh, obtendose um material em forma de pó. Geralmente, pós com granulometria entre 25 e 100 mesh são utilizados para uniformizar as partículas, pois a presença de partículas muito pequenas com outras relativamente grandes pode resultar em uma transferência de calor de forma não homogênea [48]. O PP foi misturado fisicamente com as amostras de AlMCM-48(x) na proporção de $50 \%$ em peso. Os experimentos de degradação de PP foram realizados em analisador Shimadzu, modelo DTG-60H, em uma faixa de temperatura de $30-700{ }^{\circ} \mathrm{C}$, vazão de ar sintético de $40 \mathrm{~mL} \min ^{-1}$ e taxas de aquecimento de 5,10 e $20{ }^{\circ} \mathrm{C} \mathrm{min}{ }^{-1}$. Para cada experimento, foram utilizadas amostras de aproximadamente $13 \mathrm{mg}$. Além disso, as respectivas curvas de conversão foram calculadas a partir das curvas termogravimétricas, usando-se a Equação (1).

$$
\alpha_{n}=\frac{m_{i}-m_{n}}{m_{i}-m_{f}} \times 100, \quad m_{i} \leq m_{n} \leq m_{f} \rightarrow 0 \leq \alpha_{n} \leq 1
$$

Onde, $\mathrm{m}_{\mathrm{n}}$ é a massa da amostra a uma dada temperatura, $\mathrm{m}_{\mathrm{i}}$ e $\mathrm{m}_{\mathrm{f}}$ referem-se aos valores no início e no final da perda de massa da amostra. $\alpha_{n}$ é a conversão da reação de degradação do PP a uma determinada temperatura. Os resultados termogravimétricos foram usados no método cinético de isoconversão proposto por Vyazovkin com a finalidade de determinar a energia de ativação aparente das reações de degradação do PP com e sem catalisador, usando equações cinéticas gerais baseadas nas recomendações do Comitê de Cinética da International Confederation for Thermal Analysis and Calorimetry (ICTAC) [49, 50].

\section{RESULTADOS E DISCUSSÃO}

\subsection{Atividade catalítica dos AIMCM-48(x)}

Os catalisadores AlMCM-48(x) obtidos pelo método modificado de síntese hidrotérmica com diferentes razões molares $\mathrm{Si} / \mathrm{Al}$ (50 e 100), usando pseudoboemita como fonte de $\mathrm{Al}$, foram caracterizados usando uma variedade de técnicas, incluindo difração de raios X (DRX), adsorção-dessorção de $\mathrm{N}_{2}$ a $-196{ }^{\circ} \mathrm{C}$, termogravimetria e termogravimetria derivada (TG/DTG), microscopia eletrônica de varredura e de transmissão (MEV/MET), espectrometria de energia dispersiva de raios X (EDX) e espectroscopia no infravermelho com transformada de Fourier (FTIR). Detalhes dos resultados de caracterização foram recentemente publicados [12], e alguns 
deles estão apresentados na Tabela 1. De um modo geral, os resultados revelaram estruturas altamente ordenadas, termicamente estáveis e os pesquisadores sugeriram a presença de espécies de Al tetraédrico na estrutura do AlMCM-48(x). Neste sentido, é importante destacar que a utilização da pseudoboemita, como uma promissora fonte de Al para a síntese desses catalisadores, levou à formação de materiais com sutis diferenças morfológicas devido à formação de um aglomerado de partículas esféricas interconectadas. Por último, os catalisadores AlMCM-48(100) e AlMCM-48(50) apresentaram isotermas de adsorção-dessorção do tipo IVa, que são características de materiais mesoporosos [51], área superficial de 1305 e $1052 \mathrm{~m}^{2} \mathrm{~g}^{-1} \mathrm{e}$ volume total de poros de 0,841 e $0,584 \mathrm{~cm}^{3} \mathrm{~g}^{-1}$, respectivamente.

Tabela 1: Quantidades teórica e experimental de $\mathrm{Al}_{2} \mathrm{O}_{3}$ e propriedades texturais dos catalisadores AlMCM-48(x) investigados neste trabalho. Todos os dados apresentados nesta tabela foram publicados por Dos Santos et al. (2021) [12].

\begin{tabular}{|c|c|c|c|c|c|c|}
\hline \multirow{2}{*}{ Material } & \multicolumn{2}{|c|}{$\mathrm{Al}_{2} \mathrm{O}_{3}(\%)$} & \multirow{2}{*}{$\begin{array}{c}\text { SBET }^{\mathrm{c}} \\
\left(\mathrm{m}^{2} \mathrm{~g}^{-1}\right)\end{array}$} & \multirow{2}{*}{$\begin{array}{c}V_{p^{d}} \\
\left(\mathrm{~cm}^{3} \mathrm{~g}^{-1}\right)\end{array}$} & \multirow{2}{*}{$\begin{array}{c}V_{\mu p}{ }^{e} \\
\left(\mathrm{~cm}^{3} g^{-1}\right)\end{array}$} & \multirow{2}{*}{$\begin{array}{l}\text { DBJH }^{f} \\
(\mathrm{~nm})\end{array}$} \\
\hline & Teórico $^{a}$ & Experimental $^{\mathbf{b}}$ & & & & \\
\hline AlMCM-48(100) & 4,81 & 3,93 & 1305 & 0,841 & 0,053 & 3,32 \\
\hline AIMCM-48(50) & 9,61 & 4,39 & 1052 & 0,584 & 0,125 & 3,00 \\
\hline
\end{tabular}

${ }^{\mathrm{a}}$ Baseado na composição da mistura de síntese; ${ }^{\mathrm{b}}$ Determinado por EDX; ${ }^{c} \mathrm{~S}_{\mathrm{BET}}$, área superficial específica calculada pelo método BET; ${ }^{\mathrm{d}} \mathrm{V}_{\mathrm{p}}$, volume total de poros calculado pelo método $\mathrm{BJH}$; ${ }^{\mathrm{e}} \mathrm{V}_{\mu \mathrm{p}}$, volume de microporos calculado pelo método t-Plot; ${ }^{\mathrm{f}}$ DвJH, diâmetro de poro calculado pelo método BJH.

Os experimentos de degradação térmica e catalítica do PP empregando os catalisadores AlMCM-48(100) e AlMCM-48(50) foram conduzidos por termogravimetria. A Figura 1 mostra as curvas TG/DTG em função da temperatura para três taxas diferentes de aquecimento $(5,10 \mathrm{e}$ $20{ }^{\circ} \mathrm{C} \min ^{-1}$ ) para as reações de degradação do PP na ausência de catalisador. Observa-se, a partir dessas curvas, que o perfil de degradação mudou para as temperaturas mais altas com o aumento da taxa de aquecimento. Os mesmos resultados foram encontrados na literatura por diferentes pesquisadores $[9,12,52-55]$. Esta mudança pode ser explicada por uma transferência de calor ineficiente do forno para a amostra, que obviamente aumenta com a taxa de aquecimento [9]. Outra possível razão por trás da mudança do perfil de degradação com a mudança na taxa de aquecimento é a baixa condutividade térmica do PP, que resulta no gradiente térmico [56]. Quando a taxa de aquecimento diminui, o gradiente térmico nas amostras da Figura 1 é menor e a temperatura é quase a mesma que a superfície externa e o núcleo interno das amostras. No entanto, para taxas de aquecimento mais altas, o gradiente térmico é maior e há uma diferença de temperatura entre a superfície externa e o núcleo interno das amostras.

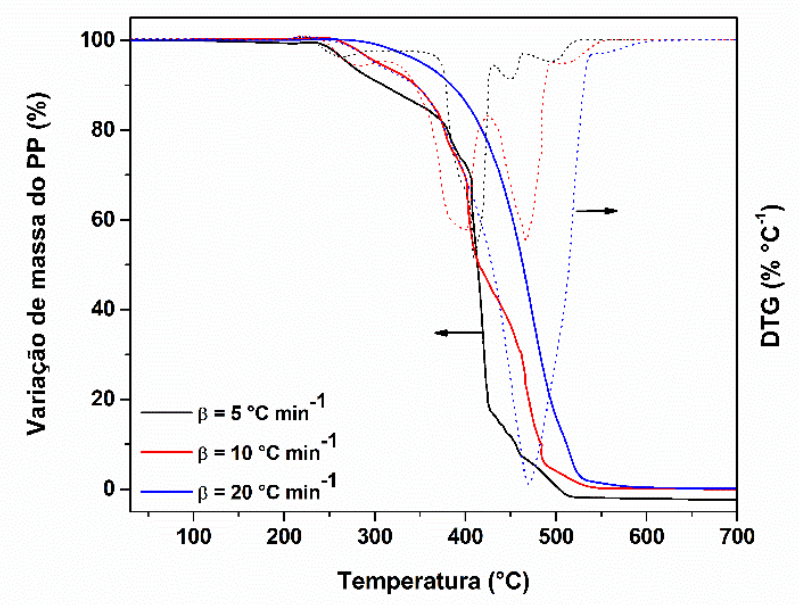

Figura 1: Perfis térmicos (TG/DTG) obtidos para a degradação do PP na ausência de catalisador a

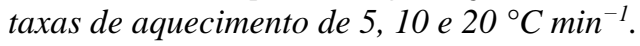


Outra característica importante observada nessas reações é a diminuição da temperatura de degradação do PP causada pela adição dos catalisadores AlMCM-48(x) (Figura 2). Alguns autores reportaram o mesmo comportamento em suas reações catalisadas de degradação de PP [40, 57-59]. Para as curvas DTG (Figura 2a) obtidas com taxa de aquecimento de $20{ }^{\circ} \mathrm{C} \mathrm{min}^{-1}$, a temperatura máxima $\left(\mathrm{T}_{\mathrm{m}}\right)$ para o PP puro foi de $471{ }^{\circ} \mathrm{C}$; após a adição do catalisador, a $\mathrm{T}_{\mathrm{m}}$ foi reduzida a 337 e $344{ }^{\circ} \mathrm{C}$ para o PP com AlMCM-48(50) e AlMCM-48(100), respectivamente. Sendo assim, as reações catalisadas apresentaram queda de temperatura em torno de $127{ }^{\circ} \mathrm{C}$ com o uso do AlMCM-48(100). Entende-se que esse comportamento pode ser explicado pela maior acessibilidade do PP aos sítios ativos do material AlMCM-48(100), o que sugere que o tamanho dos poros é um fator importante nessas reações [12]. Nos resultados experimentais divulgados por Park et al. (2008) [40], verificaram-se reduções de 140 e $155^{\circ} \mathrm{C}$ na temperatura das reações de degradação de PP com AlMCM-48 nas razões Si/Al 60 e 30, respectivamente. De acordo com Sakata et al. (1999) [60], a eficiência desses catalisadores na degradação do PP pode ser compreendida considerando-se que as ramificações e pontas da cadeia polimérica podem penetrar nos poros do catalisador, onde ocorre a reação de degradação. Consequentemente, o efeito de um catalisador deve ser mais pronunciado quando o acesso das extremidades das cadeias aos poros for facilitado. Nesse sentido, a Figura $2 \mathrm{~b}$ mostra as curvas TG para o PP puro e para o PP com 50\% em massa de AlMCM-48(x), e observa-se que o efeito do catalisador é maior para o AlMCM-48(100), com volume total de poros de $0,841 \mathrm{~cm}^{3} \mathrm{~g}^{-1}$, do que para o AlMCM-48(50), que apresenta volume total de poros consideravelmente menor $\left(0,584 \mathrm{~cm}^{3} \mathrm{~g}^{-1}\right)$.



(a)

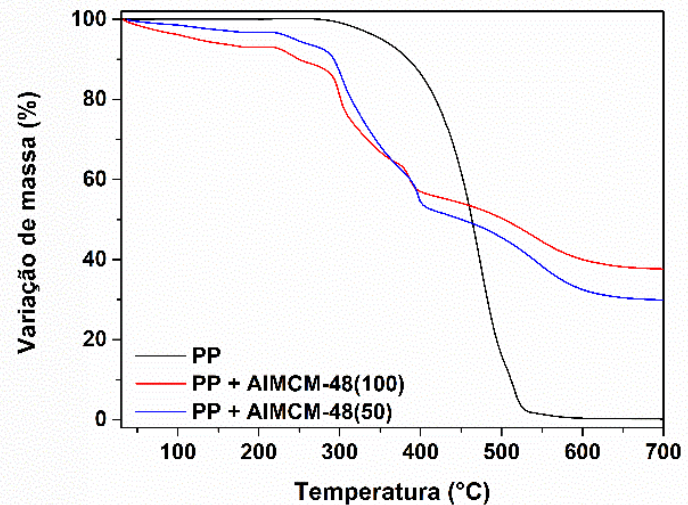

(b)

Figura 2: Perfis térmicos obtidos da degradação do PP puro e do PP com AlMCM-48(x) em diferentes razões molares de Si/Al sob taxa de aquecimento de $20{ }^{\circ} \mathrm{C} \mathrm{min}{ }^{-1}$ : (a) gráficos obtidos da DTG, (b) gráficos obtidos da TG.

A Tabela 2 mostra as temperaturas máximas $\left(\mathrm{T}_{\mathrm{m}}\right)$ da degradação do PP para as três diferentes taxas de aquecimento estudadas, mostrando a eficácia dos catalisadores nas reações. A taxa de aquecimento afetou consideravelmente o comportamento das curvas TG e DTG: quanto maior a taxa de aquecimento, maior a temperatura máxima. Este aumento de temperatura para diferentes taxas pode ser explicado pelo aumento da quantidade de energia fornecida na forma de calor, pelo mesmo incremento de tempo [61]. De maneira geral, os resultados indicaram que, com o aumento da taxa de aquecimento, ocorre também um deslocamento das temperaturas médias de decomposição do PP para valores mais elevados. Resultados semelhantes também foram relatados na literatura $[11,39,41,52,62,63]$ mostrando que esse comportamento provavelmente tem relação com o tamanho das macromoléculas de PP que inicialmente sofrem reações na superfície do catalisador e posteriormente se difundem através dos poros das amostras de catalisador em reações secundárias, após a formação de fragmentos menores. No estágio inicial, a reação pode ser submetida à ocorrência de limitações de difusão ao transporte 
de massa que causam diminuição nas taxas globais de reação superficial. Além disso, estudos sobre a pirólise catalítica de polímeros e outros materiais têm mostrado que o desempenho do catalisador é importante tanto na redução das temperaturas de degradação quanto na seletividade a produtos de alto valor agregado $[9,13,39-41,52,64,65]$.

Tabela 2: Valores termogravimétricos obtidos da degradação do PP puro e do PP com AlMCM-48(x) investigados neste trabalho sob diferentes taxas de aquecimento $(\beta)$.

\begin{tabular}{|c|c|c|c|}
\hline \multirow{2}{*}{ Amostra } & \multicolumn{3}{|c|}{$\mathbf{T}_{\mathbf{m}}{ }^{\mathbf{a}}\left({ }^{\circ} \mathbf{C}\right)$} \\
\hline & $\beta=5\left({ }^{\circ} \mathrm{C} \mathrm{min}^{-1}\right)$ & $\beta=10\left({ }^{\circ} \mathrm{C} \mathrm{min}{ }^{-1}\right)$ & $\beta=20\left({ }^{\circ} \mathrm{C} \mathrm{min}-1\right)$ \\
\hline PP & 410 & 467 & 471 \\
\hline$P P+A I M C M-48(100)$ & 333 & 336 & 344 \\
\hline$P P+A I M C M-48(50)$ & 332 & 331 & 337 \\
\hline
\end{tabular}

${ }^{a} \mathrm{~T}_{\mathrm{m}}$, temperatura máxima obtidas das curvas DTG.

As perdas de massa registradas a partir das curvas termogravimétricas (Figura 3, gráficos obtidos da degradação do PP com 50\% em massa de AlMCM-48(x)) foram transformadas em diferentes valores de conversão. Considerando-se que a perda de massa total condiz a 100\% de conversão, as perdas de massa em temperaturas menores foram normalizadas em relação à perda de massa total, originando as curvas de conversão [9]. A Figura 3 mostra um conjunto de curvas de conversão obtidas com diferentes taxas de aquecimento: 5,10 e $20{ }^{\circ} \mathrm{C} \min ^{-1}$, onde se observa que as amostras contendo AlMCM-48(x) apresentaram temperaturas mais baixas para degradação do PP, o que sugere que a conversão também foi significativamente influenciada pela temperatura de degradação e pela taxa de aquecimento. Além disso, os dados na Tabela 3 indicaram que a conversão do PP apresenta valores de 67 a 69\% com o AlMCM-48(x) para taxa de aquecimento de $5{ }^{\circ} \mathrm{C} \mathrm{min}{ }^{-1}$; quando a taxa de aquecimento é de $10{ }^{\circ} \mathrm{C} \mathrm{min}{ }^{-1}$, a conversão foi em torno de 58\% (Tabela 3), e com uma taxa de aquecimento de $20{ }^{\circ} \mathrm{C} \mathrm{min}^{-1}$, a conversão apresentou valores de 55 e 52\% com AlMCM-48(100) e AlMCM-48(50), respectivamente (Tabela 3). Para a taxa de aquecimento mais baixa $\left(5^{\circ} \mathrm{C} \mathrm{min}{ }^{-1}\right)$, os catalisadores AlMCM-48(x) apresentaram valores de conversão mais elevados (67-69\%) e, em contraste, para a taxa de aquecimento de $20{ }^{\circ} \mathrm{C} \mathrm{min}^{-1}$ registrou-se um comportamento inverso, uma diferença de conversão de 12-17\% com AlMCM-48(100) e AlMCM-48(50), respectivamente, sugerindo que uma limitação difusional pode ocorrer nos microporos do catalisador [11]. Comportamento semelhante foi apresentado por Dos Santos et al. (2021) [12] em reações de degradação de polietileno sob AlMCM-48.

Tabela 3: Valores de conversão obtidos para a degradação do PP investigados neste trabalho sob diferentes taxas de aquecimento $(\beta)$.

\begin{tabular}{cccc}
\hline \multirow{2}{*}{ Amostra } & \multicolumn{3}{c}{ Conversão ${ }^{\mathbf{a}}(\boldsymbol{\%})$} \\
\cline { 2 - 4 } & $\boldsymbol{\beta}=\mathbf{5}\left({ }^{\circ} \mathbf{C} \mathbf{~ m i n}^{-1}\right)$ & $\boldsymbol{\beta}=\mathbf{1 0}\left({ }^{\circ} \mathbf{C}\right.$ min $\left.^{-1}\right)$ & $\boldsymbol{\beta}=\mathbf{2 0}\left({ }^{\circ} \mathbf{C} \mathbf{~ m i n}^{-\mathbf{1}}\right)$ \\
\hline PP & 100 & 100 & 100 \\
PP + AlMCM-48(100) & 67 & 58 & 55 \\
PP + AIMCM-48(50) & 69 & 58 & 52 \\
\hline
\end{tabular}

a Valores de conversão obtidos pela Equação (1) e normalizados em relação à perda de massa total, de acordo com Silva et al. (2017) [9]. 


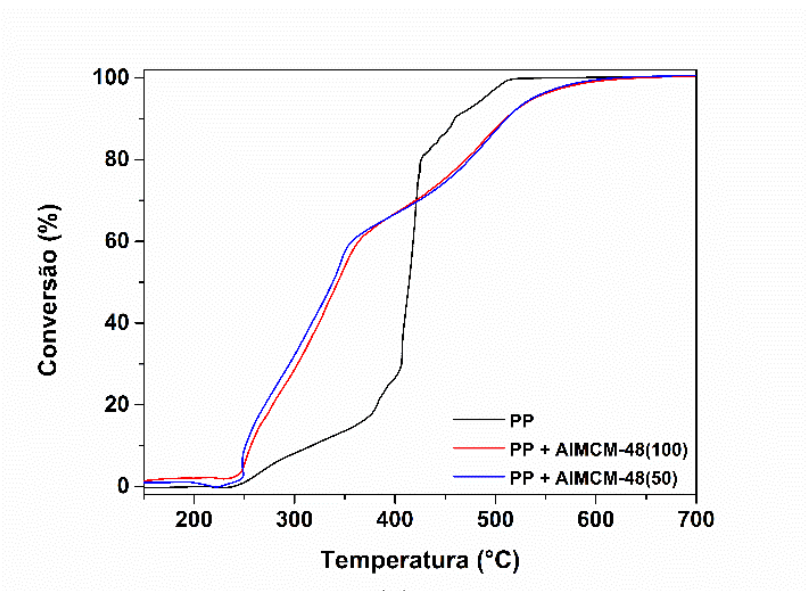

(a)

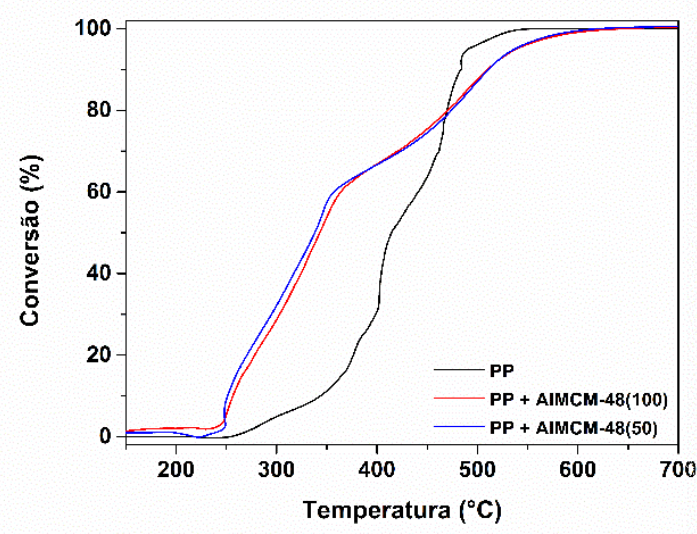

(b)

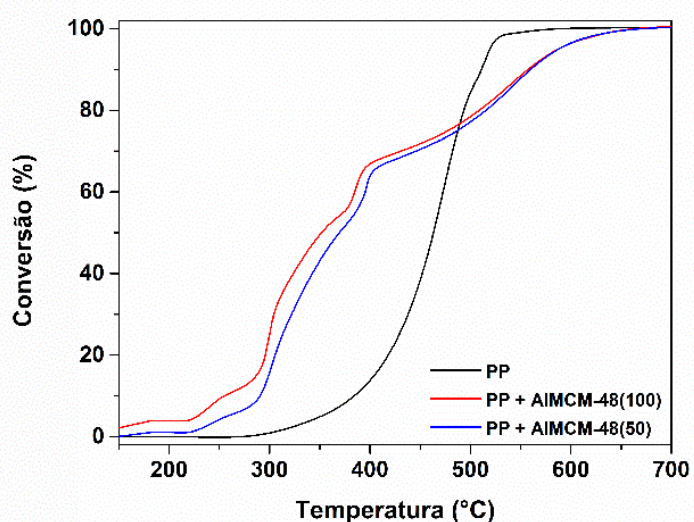

(c)

Figura 3: Curvas de conversão obtidas para a degradação do PP puro e do PP com AlMCM-48(x) em diferentes razões molares de Si/Al sob diferentes taxas de aquecimento em (a), (b) e (c) correspondendo a 5,10 e $20{ }^{\circ} \mathrm{C} \mathrm{min}^{-1}$, respectivamente.

\subsection{Análise cinética via modelo de Vyazovkin}

A velocidade de reação de um processo catalítico depende da conversão $(\alpha)$, da temperatura (T) e do tempo (t). Para cada processo, a velocidade de reação dada como função da conversão $\mathrm{f}(\alpha)$ é diferente e pode ser determinada a partir de dados experimentais. Para reações complexas, como a degradação de plásticos, a função $f(\alpha)$ é complicada e, em geral, desconhecida [66]. Aplicando-se o método de isoconversão sugerido por Vyazovkin, podem ser obtidos dados confiáveis e informações cinéticas consistentes sobre o processo como um todo [49, 50]. Assim, a cinética de degradação térmica e catalítica do PP tem como ideia que a variação da sua massa, em decorrência de uma transformação de temperatura em função do tempo, segue a Equação (2) [67-70].

$$
\frac{d \alpha}{d t}=k(T) f(\alpha)
$$

Em que, $\alpha$ é o grau de conversão da amostra calculado de acordo com a Equação (1) e d $\alpha / d t$ representa a taxa de reação; $f(\alpha)$ é a função que retrata o modelo de degradação térmica para $o$ processo em estudo e $\mathrm{k}(\mathrm{T})$ é a constante da taxa de degradação dependente da temperatura, que pode ser expressa pela equação de Arrhenius, conforme a Equação (3). 


$$
k(T)=A e^{\left(-E_{a} / R T\right)}
$$

Onde A é o fator pré-exponencial; $E_{a}$ é a energia de ativação aparente da reação de degradação; $\mathrm{R}$ é a constante universal dos gases ideais e $\mathrm{T}$, a temperatura absoluta. A substituição da Equação (3) na Equação (2) resulta na Equação (4).

$$
\frac{d \alpha}{d t}=A e^{\left(-E_{a} / R T\right)} f(\alpha)
$$

A Equação (4) pode ser considerada uma expressão geral para condições isotérmicas. No caso de condições não isotérmicas, onde a amostra é aquecida com uma taxa de aquecimento constante, tal equação pode ser transformada em outra que descreve a taxa de reação de degradação em função da temperatura e, em seguida, pode ser expressa sob a forma da Equação (5) em que $\beta$ é a taxa de aquecimento.

$$
\frac{d \alpha}{d T}=\frac{A}{\beta} e^{\left(-E_{a} / R T\right)} f(\alpha)
$$

Os parâmetros cinéticos $\left(\mathrm{A}\right.$ e $\left.\mathrm{E}_{\mathrm{a}}\right)$ dos dados termogravimétricos podem ser determinados por diferentes métodos (model-fitting e model-free kinetics), que envolvem procedimentos de integração, diferenciação ou aproximação para resolver as Equações (4) e (5) [49, 50]. Os métodos de model-fitting permitem o ajuste de vários modelos às curvas de conversão em função da temperatura e a determinação simultânea da energia de ativação e do fator de frequência. A abordagem model-free kinetics permite estimar os valores da energia de ativação em função da conversão sem a suposição de um modelo cinético $(\mathrm{f}(\alpha))$. Por exemplo, a variação na energia de ativação com a conversão indica que o processo é complexo (reação em várias etapas); por outro lado, se as energias de ativação não dependem da conversão, o processo é considerado simples (reação em uma única etapa) [71].

O método de Vyazovkin [46, 49, 50] é um exemplo bem conhecido de abordagens do tipo model-free kinetics, que envolve uma aproximação da Equação (6), permitindo a variação isotérmica da taxa de aquecimento $(\beta)$. Este método é isoconversional e pode ser aplicado para calcular a energia de ativação de uma dada reação química em função da temperatura, a partir de dados de perda de massa em diferentes taxas de aquecimento e sem a necessidade de um modelo de taxa de reação em função das concentrações dos reagentes. A Equação (6) representa a forma integrada do modelo cinético proposto por Vyazovkin, onde $g(\alpha)$ (a forma integral do modelo cinético, ou seja, $\mathrm{g}(\alpha)=\mathrm{kt}$ ) é uma relação em função da conversão [70]. Detalhes deste método são relatados na literatura [72].

$$
\ln \left(\frac{\beta}{T_{\alpha}^{2}}\right)=\ln \left[\frac{A R}{g_{(\alpha)} E_{a}}\right]-\frac{E_{a}}{R T_{\alpha}}
$$

Onde $\alpha$ é a conversão da reação de degradação do polímero; $\mathrm{T}_{\alpha}$ é a temperatura para alcançar a conversão $\alpha ; \beta$ é a taxa de aquecimento; $\mathrm{R}$ é a constante universal dos gases; A é o fator préexponencial de Arrhenius; $E_{a}$ é a energia de ativação para uma determinada conversão $\alpha$ e g $(\alpha)$ é a integral do modelo cinético da taxa de reação em função da conversão.

Na maioria dos casos, a função $g(\alpha)$ não apresenta uma forma definida e, após o ajuste do modelo, $g(\alpha)$ torna-se implícito no coeficiente linear obtido da Equação (6), indicando que o gráfico de $\ln \left(\beta / \mathrm{T}^{2}\right)$ em função de $1 / \mathrm{T}$ produz uma linha reta e, de sua inclinação, pode ser determinada a energia de ativação. Esta energia de ativação calculada é chamada de energia de ativação aparente porque é a soma das energias de ativação de reações químicas e processos físicos que ocorrem durante a conversão [73]. A grande vantagem do método proposto por Vyazovkin é que os valores de $\mathrm{E}_{\mathrm{a}}$ são facilmente obtidos sem o conhecimento do mecanismo de controle da reação [72]. 
As perdas de massa determinadas pelas curvas TG foram transformadas em dados de conversão, como apresentados na Figura 3, e submetidas ao tratamento cinético pelo método de Vyazovkin. Considerando-se que a perda de massa total corresponde a $100 \%$ da conversão, as perdas de massa em temperaturas mais baixas foram normalizadas para a perda de massa total. Os valores de conversão na temperatura da taxa de degradação máxima, listados na Tabela 3, geralmente são aceitos como parâmetros decisivos para eficácia do método cinético [74]. A Figura 4 apresenta os gráficos do $\ln \left(\beta / T^{2}\right)$ em função de $1 / T$ produzindo linhas retas, cuja inclinação é proporcional à energia de ativação. Sendo assim, as reações que têm energias de ativação baixas apresentam gráficos de Arrhenius pouco inclinados, ou seja, apresentam velocidades que aumentam sutilmente com a temperatura. Por conseguinte, as reações que têm energias de ativação altas apresentam gráficos de Arrhenius muito inclinados e têm velocidades que dependem fortemente da temperatura.

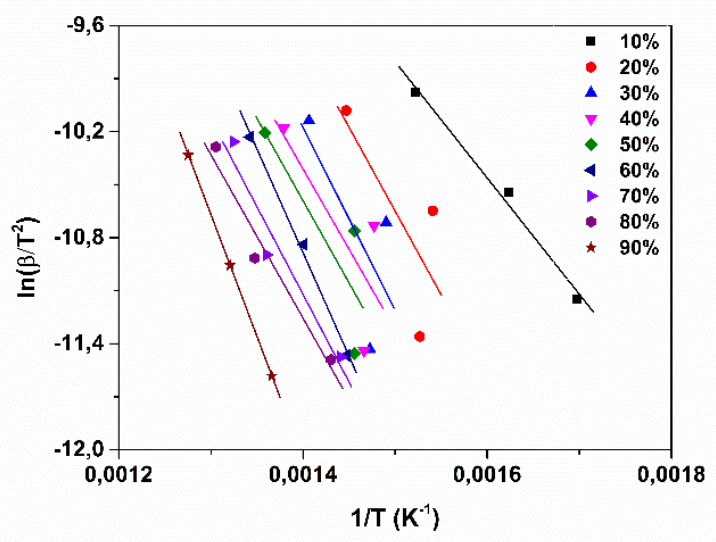

(a)

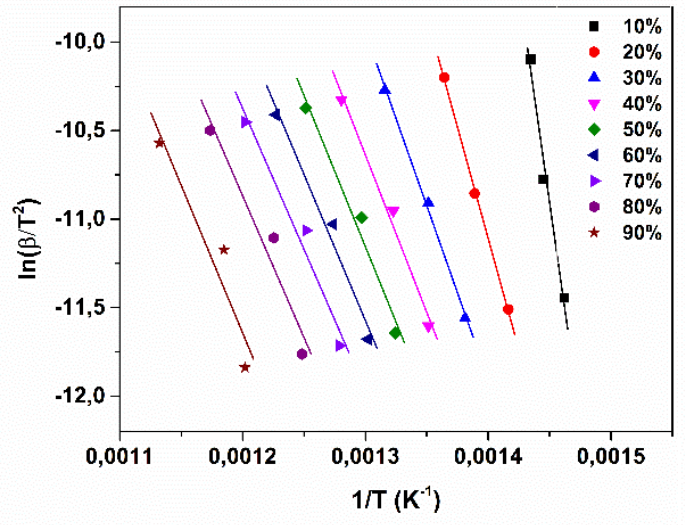

(b)

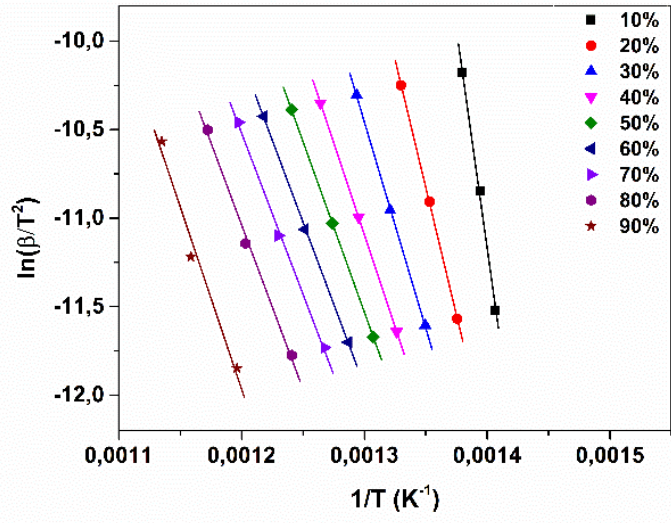

(c)

Figura 4: Gráficos do modelo de Vyazovkin para diferentes conversões ( $\alpha$ ): (a) amostra de PP puro; (b) amostra de PP + AlMCM-48(100) e (c) amostras de PP + AlMCM-48(50).

Para cada valor de conversão, a $E_{a}$ foi calculada a partir da inclinação das linhas de regressão, através da média de diferentes taxas de aquecimento. O gráfico da Figura 5 mostra os valores da $\mathrm{E}_{\mathrm{a}}$ média em função da conversão para a degradação do $\mathrm{PP}$ puro e do $\mathrm{PP}+50 \%$ em massa dos catalisadores AlMCM-48(x). As amostras de PP + AlMCM-48(100) e AlMCM48(50) apresentaram valores médios de $\mathrm{E}_{\mathrm{a}}$ de 59 e $66 \mathrm{~kJ} \mathrm{~mol}^{-1}$, respectivamente, em contraste a $89 \mathrm{~kJ} \mathrm{~mol}^{-1}$ do PP puro. Consequentemente, a adição do catalisador resultou em uma redução nos valores médios da $\mathrm{E}_{\mathrm{a}}$ em relação ao PP puro, evidenciando influência do sistema de poros dos catalisadores nas reações de degradação, que é um parâmetro importante para o desempenho catalítico desses materiais na degradação do PP em termos de efeitos difusionais [44], o que 
pode corroborar o resultado mais expressivo do AlMCM-48(100), que teve uma diminuição média de $59 \mathrm{~kJ} \mathrm{~mol}^{-1}$ comparado com o PP puro. Park et al. (2008) [40] apresentaram em seus experimentos de degradação de PP sob AlMCM-48 resultados semelhantes de 102 e $96 \mathrm{~kJ} \mathrm{~mol}^{-1}$ com o uso dos catalisadores nas razões Si/Al de 60 e 30, respectivamente. Na Figura 5, observase ainda uma redução nas energias de ativação em baixo e alto grau de conversão seguido por um comportamento cinético quase constante dos catalisadores AlMCM-48(x), sem a presença de múltiplas bandas, especificamente na faixa de conversão de $20 \leq \alpha \leq 90 \%$.

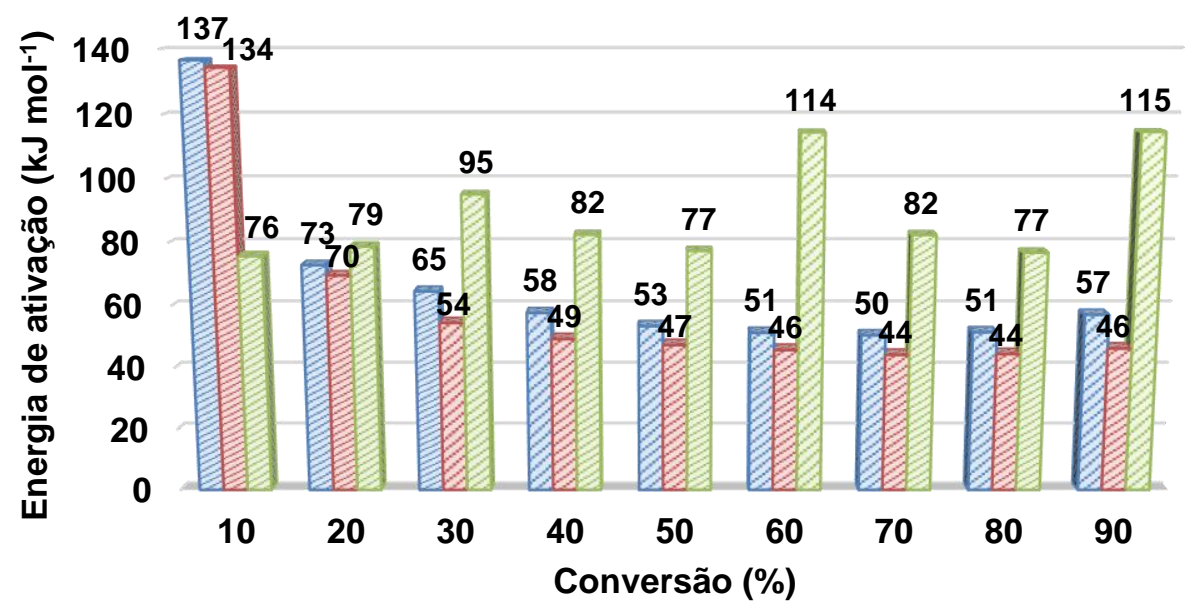

$₫ P P+A I M C M-48(50) \quad \triangle P P+A I M C M-48(100) \quad \triangle P P$ puro

Figura 5: Comportamento cinético sobre a $E_{a}$ em função da $\alpha$ das amostras de PP puro (E $E_{a}$ média de 89 $\mathrm{kJ} \mathrm{mol}^{-1}$ ) e do PP sobre diferentes catalisadores AlMCM-48(100) (Ea média de $59 \mathrm{~kJ} \mathrm{~mol}^{-1}$ ) e AlMCM48(50) ( $E_{a}$ média de $66 \mathrm{~kJ} \mathrm{~mol}^{-1}$ ), de acordo com o método cinético de Vyazovkin.

Em concordância com os valores obtidos, pode-se observar que o processo de degradação térmica do PP provavelmente consistiu em muitos processos complexos, como cisão de cadeias aleatórias, transferência de radical, etc., que ocorreram simultaneamente durante a degradação. Essas etapas de quebra de ligação têm suas taxas e energias de ativação individuais, e a cinética termogravimétrica é capaz de medir a taxa geral de degradação e a energia de ativação aparente do processo da degradação catalítica do PP. Sendo assim, a degradação catalítica torna-se menos complexa ao se discutir a taxa de degradação em função das propriedades estruturais dos catalisadores, sabendo-se que a atividade do AlMCM-48(x) é baseada na formação de intermediários reativos capazes de reduzir a energia de ativação das reações parciais correspondentes como evidenciado nas conversões acima de 30\% (Figura 5) [10].

\section{CONCLUSÃ̃}

O estudo termogravimétrico da degradação catalítica do PP mostrou que a adição de AlMCM-48(x) em diferentes razões molares Si/Al (50 e 100) foi responsável por uma redução considerável de $77-136{ }^{\circ} \mathrm{C}$ ao comparar com a reação sem catalisador, com referência à temperatura da taxa de decomposição máxima $\left(\mathrm{T}_{\mathrm{m}}\right)$. Isto indicou a potencial atividade catalítica desses materiais, pois as reações iniciaram em temperaturas bem abaixo nos processos catalíticos do que nos processos não catalíticos. Além disso, curvas de conversão do PP e do PP na presença dos catalisadores AlMCM-48(x) foram obtidas, e foi possível comparar as condições operacionais para se obter uma determinada conversão em função da temperatura em três taxas de aquecimento: 5,10 e $20{ }^{\circ} \mathrm{C} \mathrm{min}^{-1}$. O desempenho da degradação do $\mathrm{PP}, \mathrm{PP}+$ AlMCM-48(50) e PP + AlMCM-48(100) com o uso do método cinético de isoconversão proposto por Vyazovkin forneceu os valores das energias de ativação aparente em função do 
grau de conversão. Contudo, o método de Vyazovkin evidenciou uma média de $\mathrm{E}_{\mathrm{a}}=89$, 66 e 59 $\mathrm{kJ} \mathrm{mol}^{-1}$ para a degradação do PP puro, PP + AlMCM-48(50) e PP + AlMCM-48(100), respectivamente. Este comportamento pôde ser explicado em decorrência da forte influência do volume de poros dos catalisadores usados nas reações de degradação do PP e, como consequência, a maior eficiência foi com o AlMCM-48(100) quando comparado com o PP puro. Em concordância com os resultados obtidos, o AlMCM-48(x) pode ser considerado um catalisador promissor para a degradação de PP e de outros resíduos plásticos.

\section{AGRADECIMENTOS}

Este trabalho teve o apoio do Conselho Nacional de Desenvolvimento Científico e Tecnológico $(\mathrm{CNPq})$ e da Coordenação de Aperfeiçoamento de Pessoal de Nível Superior (CAPES) - Código financeiro 001. Além disso, os autores agradecem ao GCAR/IQB/UFAL, LabCat/UFS e LSCAT/CTEC/UFAL.

\section{REFERÊNCIAS BIBLIOGRÁFICAS}

1. Bertin D, Leblanc M, Marque SRA, Siri D. Polypropylene degradation: Theoretical and experimental investigations. Polym Degrad Stab. 2010;95(5):782-91. doi: 10.1016/j.polymdegradstab.2010.02.006

2. Zhang K, Hamidian AH, Tubić A, Zhang Y, Fang JKH, Wu C, et al. Understanding plastic degradation and microplastic formation in the environment: A review. Environ Pollut. 2021 Jan;274:116554. doi: 10.1016/j.envpol.2021.116554

3. Ceresana. Polypropylene market report [Internet]. 5. ed. Alemanha: Ceresana; 2019 [citado em 29 mar 2021]. Disponível em: https://www.ceresana.com/en/market-studies/plastics/polypropylene/

4. Barra R, Leonard SA. Plastics and the circular economy [Internet]. 54th Global Environment Facility Council Meeting; Da Nang, Vietnã, 24-26 Jun 2018. Disponível em: https://www.thegef.org/councilmeeting-documents/plastics-and-circular-economy

5. Mrowiec B. Plastics in the circular economy (CE). Ochr Środowiska i Zasobów Nat. 2018;29(4):16-9. doi: 10.2478/oszn-2018-0017

6. Geyer R, Jambeck JR, Law KL. Production, use, and fate of all plastics ever made. Sci Adv. 2017 Jul;3(7):e1700782. doi: 10.1126/sciadv.1700782

7. Chamas A, Moon H, Zheng J, Qiu Y, Tabassum T, Jang JH, et al. Degradation rates of plastics in the environment. ACS Sustain Chem Eng. 2020 Feb;8(9):3494-11. doi: 10.1021/acssuschemeng.9b06635

8. Webb HK, Arnott J, Crawford RJ, Ivanova EP. Plastic degradation and its environmental implications with special reference to poly(ethylene terephthalate). Polymers. 2013 Dec;5(1):1-18. doi: 10.3390/polym5010001

9. Silva AOS, Souza MJB, Pedrosa AMG, Coriolano ACF, Fernandes VJ, Araujo AS. Development of HZSM-12 zeolite for catalytic degradation of high-density polyethylene. Microporous Mesoporous Mater. 2017 Feb;244:1-6. doi: 10.1016/j.micromeso.2017.02.049

10. Silva BJB, Sousa LV, Sarmento LRA, Alencar SL, Quintela PHL, Silva AOS. Kinetic study of thermocatalytic degradation of UHMWPE over microporous and hierarchical ZSM-23 zeolite. Appl Catal B Environ. 2020 Jan;267:118699. doi: 10.1016/j.apcatb.2020.118699

11. Souza MJB, Silva THA, Ribeiro TRS, da Silva AOS, Pedrosa AMG. Thermal and catalytic pyrolysis of polyvinyl chloride using micro/mesoporous ZSM-35/MCM-41 catalysts. J Therm Anal Calorim. 2020 Sep;140(1):167-75. doi: 10.1007/s10973-019-08803-7

12. Dos Santos TG, Silva AOS, Pedrosa AMG, Araujo AS, Souza MJB. Structure and catalytic activity of highly ordered AlMCM-48 materials with different $\mathrm{Si} / \mathrm{Al}$ ratios on the degradation of high-density polyethylene. J Porous Mater. 2021 Feb;28:919-28. doi: 10.1007/s10934-021-01044-w

13. Kaminsky W, Zorriqueta IJN. Catalytical and thermal pyrolysis of polyolefins. J Anal Appl Pyrolysis. 2007 Dec;79:368-74. doi:10.1016/j.jaap.2006.11.005

14. García RA, Serrano DP, Otero D. Catalytic cracking of HDPE over hybrid zeolitic-mesoporous materials. J Anal Appl Pyrolysis. 2005 Jan;74(1-2):379-86. doi: 10.1016/j.jaap.2004.11.002

15. Escola JM, Aguado J, Serrano DP, García A, Peral A, Briones L, et al. Catalytic hydroreforming of the polyethylene thermal cracking oil over $\mathrm{Ni}$ supported hierarchical zeolites and mesostructured aluminosilicates. Appl Catal B Environ. 2011 Jun;106(3-4):405-15. doi: 10.1016/j.apcatb.2011.05.048

16. Ohkita H, Nishiyama R, Tochihara Y, Mizushima T, Kakuta N, Morioka Y, et al. Acid properties of silica-alumina catalysts and catalytic degradation of polyethylene. Ind Eng Chem Res. 1993 Dec;32(12):3112-6. doi: 10.1021/ie00024a021 
17. Aguado J, Sotelo JL, Serrano DP, Calles JA, Escola JM. Catalytic conversion of polyolefins into liquid fuels over MCM-41: Comparison with ZSM-5 and amorphous $\mathrm{SiO}_{2}-\mathrm{Al}_{2} \mathrm{O}_{3}$. Energy Fuels. 1997 Apr;11(6):1225-31. doi: 10.1021/ef970055v

18. Vyas A, Singh SK, Sharma K, Singh S. Catalytic cracking of waste polypropylene in a Nitrogen fluidized bed reactor. J Geosci Environ Prot. 2016 Apr;4:20-5. doi: 10.4236/gep.2016.44004

19. Marcilla A, Gómez-Siurana A, Berenguer D. Study of the influence of the characteristics of different acid solids in the catalytic pyrolysis of different polymers. Appl Catal A Gen. 2006 Jan;301(2):22231. doi: 10.1016/j.apcata.2005.12.018

20. Vartuli JC, Schmitt KD, Kresge CT, Roth WJ, Leonowicz ME, McCullen SB, et al. Effect of surfactant/silica molar ratios on the formation of mesoporous molecular sieves: Inorganic mimicry of surfactant liquid-crystal phases and mechanistic implications. Chem Mater. 1994 Aug;6(12):2317-26. doi: $10.1021 / \mathrm{cm} 00048 \mathrm{a} 018$

21. Yates TJV, Thomas JM, Fernandez JJ, Terasaki O, Ryoo R, Midgley PA. Three-dimensional realspace crystallography of MCM-48 mesoporous silica revealed by scanning transmission electron tomography. Chem Phys Lett. 2006 Dec;418(4-6):540-3. doi: 10.1016/j.cplett.2005.11.031

22. Kim SN, Son WJ, Choi JS, Ahn WS. $\mathrm{CO}_{2}$ adsorption using amine-functionalized mesoporous silica prepared via anionic surfactant-mediated synthesis. Microporous Mesoporous Mater. 2008 Mar;115(3):497-503. doi: 10.1016/j.micromeso.2008.02.025

23. Chen F, Huang L, Yang X, Wang Z. Synthesis of Al-substituted MCM-41 and MCM-48 solid acids with mixed cationic-anionic surfactants as templates. Mater Lett. 2013 Jul;109:299-301. doi: 10.1016/j.matlet.2013.07.079

24. Yin LJ, Chen DZ, Wang H, Ma XB, Zhou GM. Simulation of an innovative reactor for waste plastics pyrolysis. Chem Eng J. 2014 Oct;237:229-35. doi: 10.1016/j.cej.2013.09.114

25. Dos Santos TG, Silva AOS, Meneghetti SMP. Comparison of the hydrothermal syntheses of Snmagadiite using $\mathrm{Na}_{2} \mathrm{SnO}_{3}$ and $\mathrm{SnCl}_{4} \cdot 5 \mathrm{H}_{2} \mathrm{O}$ as the precursors. Appl Clay Sci. 2019 Aug;183:105293. doi: 10.1016/j.clay.2019.105293

26. Russo PA, Ribeiro Carrott MML, Carrott PJM, Lopes JM, Ramôa Ribeiro F, Rocha J. Structure and catalytic activity of Al-MCM-48 materials synthesised at room temperature: Influence of the aluminium source and calcination conditions. Microporous Mesoporous Mater. 2008 Jan;114(13):293-302. doi: 10.1016/j.micromeso.2008.01.015

27. Ribeiro Carrott MML, Conceição FL, Lopes JM, Carrott PJM, Bernardes C, Rocha J, et al. Comparative study of Al-MCM materials prepared at room temperature with different aluminium sources and by some hydrothermal methods. Microporous Mesoporous Mater. 2006 Mar;92(1-3):27085. doi: 10.1016/j.micromeso.2006.01.010

28. Dapurkar SE, Selvam P. Mesoporous H-AlMCM-48: Highly efficient solid acid catalyst. Appl Catal A Gen. 2003 Apr;254(2):239-49. doi: 10.1016/S0926-860X(03)00486-1

29. Hartmann M, Bischof C. Synthesis and characterization of aluminum-containing MCM-48. Stud Surf Sci Catal. 1998 Sep;117:249-56. doi: 10.1016/S0167-2991(98)80999-3

30. Øye G, Sjöblom J, Stöcker M. Synthesis and characterization of siliceous and aluminum-containing mesoporous materials from different surfactant solutions. Microporous Mesoporous Mater. 1999 Feb;27(2-3):171-80. doi: 10.1016/S1387-1811(98)00251-0

31. Xia Y, Mokaya R. Aluminosilicate MCM-48 materials with enhanced stability via simple postsynthesis treatment in water. Microporous Mesoporous Mater. 2004 Sep;68(1-3):1-10. doi: 10.1016/j.micromeso.2003.09.029

32. Huang L, Huang Q, Xiao H, Eic M. Al-MCM-48 as a potential hydrotreating catalyst support: I Synthesis and adsorption study. Microporous Mesoporous Mater. 2008 Aug;111(1-3):404-10. doi: 10.1016/j.micromeso.2007.08.011

33. Eimer GA, Pierella LB, Monti GA, Anunziata OA. Synthesis and characterization of Al-MCM-41 and Al-MCM-48 mesoporous materials. Catal Letters. 2002 Mar;78(1-4):65-75. doi: 10.1023/A:1014924332500

34. Chang JC, Ko AN. Novel synthesis of $\varepsilon$-caprolactam from cyclohexanone-oxime via beckmann rearrangement over mesoporous molecular sieves MCM-48. Catal Today. 2004 Sep;97(4):241-47. doi: 10.1016/j.cattod.2004.07.010

35. Krithiga T, Vinu A, Ariga K, Arabindoo B, Palanichamy M, Murugesan V. Selective formation 2,6diisopropyl naphthalene over mesoporous Al-MCM-48 catalysts. J Mol Catal A Chem. 2005 Jun;237(1-2):238-45. doi: 10.1016/j.molcata.2005.05.008

36. Venkatachalam K, Visuvamithiran P, Sundaravel B, Palanichamy M, Murugesan V. Catalytic performance of Al-MCM-48 molecular sieves for isopropylation of phenol with isopropyl acetate. Chinese J Catal. 2012 Nov;33(3):478-86. doi: 10.1016/S1872-2067(11)60339-2 
37. Wang K, Lin Y, Morris MA, Holmes JD. Preparation of MCM-48 materials with enhanced hydrothermal stability. J Mater Chem. 2006 Aug;16(41):4051-7. doi: 10.1039/b607599a

38. Elfadly AM, Zeid IF, Yehia FZ, Rabie AM, Aboualala MM, Park SE. Highly selective BTX from catalytic fast pyrolysis of lignin over supported mesoporous silica. Int J Biol Macromol. 2016 May;91:278-93. doi: 10.1016/j.ijbiomac.2016.05.053

39. Jeon JK, Park HJ, Yim JH, Kim JM, Jung J, Park YK. Catalytic cracking of LLDPE over MCM-48. Solid State Phenom. 2007 Jun;124-126:1757-60. doi: 10.4028/www.scientific.net/SSP.124-126.1757

40. Park HJ, Yim JH, Jeon JK, Man Kim J, Yoo KS, Park YK. Pyrolysis of polypropylene over mesoporous MCM-48 material. J Phys Chem Solids. 2008 May-Jun;69(5-6):1125-8. doi: 10.1016/j.jpcs.2007.10.083

41. Park YK, Lee HW, Lee JY, Kim YM. The use of high density polyethylene (HDPE) as a co-feeding feedstock on the catalytic pyrolysis of yellow poplar over Al-MCM-48 and Al-MSU-F. J Anal Appl Pyrolysis. 2018 Aug;135:390-6. doi: 10.1016/j.jaap.2018.08.008

42. Noroozi M, Panahi-Sarmad M, Abrisham M, Amirkiai A, Asghari N, Golbaten-Mofrad H, et al. Nanostructure of aerogels and their applications in thermal energy insulation. ACS Appl Energy Mater. 2019 Jul;2(8):5319-49. doi: 10.1021/acsaem.9b01157

43. Noroozi M, Panahi-Sarmad M, Bahramian AR. Thermal insulation behavior of functionally graded aerogel: The role of novolac molecular-weight. Polymer. 2019 Jun;178:121575. doi: 10.1016/j.polymer.2019.121575

44. Pacheco Filho JGA, Graciliano EC, Silva AOS, Souza MJB, Araujo AS. Thermo gravimetric kinetics of polypropylene degradation on ZSM-12 and ZSM-5 catalysts. Catal Today. 2005 Aug;107-108:50712. doi: 10.1016/j.cattod.2005.07.065

45. Çepelioğullar, Haykiri-Açma H, Yaman S. Kinetic modelling of RDF pyrolysis: Model-fitting and model-free approaches. Waste Manag. 2016 Nov;48:275-84. doi: 10.1016/j.wasman.2015.11.027

46. Vyazovkin S, Wight CA. Model-free and model-fitting approaches to kinetic analysis of isothermal and nonisothermal data. Thermochim Acta. 1999 Dec;340-341:53-68. doi: 10.1016/S00406031(99)00253-1

47. Xu F, Wang B, Yang D, Hao J, Qiao Y, Tian Y. Thermal degradation of typical plastics under high heating rate conditions by TG-FTIR: Pyrolysis behaviors and kinetic analysis. Energy Convers Manag. 2018 Jun;171:1106-15. doi: 10.1016/j.enconman.2018.06.047

48. Spinacé MAS, De Paoli MA. A tecnologia da reciclagem de polímeros. Quim Nova. 2005 JanFeb;28(1):65-72. doi: 10.1590/S0100-40422005000100014

49. Vyazovkin S, Burnham AK, Criado JM, Pérez-Maqueda LA, Popescu C, Sbirrazzuoli N. ICTAC Kinetics Committee recommendations for performing kinetic computations on thermal analysis data. Thermochim Acta. 2011 Jun;520(1-2):1-19. doi: 10.1016/j.tca.2011.03.034

50. Vyazovkin S, Chrissafis K, Di Lorenzo ML, Koga N, Pijolat M, Roduit B, et al. ICTAC Kinetics Committee recommendations for collecting experimental thermal analysis data for kinetic computations. Thermochim Acta. 2014 Aug;590:1-23. doi: 10.1016/j.tca.2014.05.036

51. Thommes M, Kaneko K, Neimark AV, Olivier JP, Rodriguez-Reinoso F, Rouquerol J, et al. Physisorption of gases, with special reference to the evaluation of surface area and pore size distribution (IUPAC Technical Report). Pure Appl Chem. 2015 Jul;87(9-10):1051-69. doi: 10.1515/pac-2014-1117

52. Zhang B, Zhong Z, Ding K, Song Z. Production of aromatic hydrocarbons from catalytic co-pyrolysis of biomass and high density polyethylene: Analytical Py-GC/MS study. Fuel. 2015 Sep;139:622-8. doi: 10.1016/j.fuel.2014.09.052

53. Ahmad I, Ismail Khan M, Ishaq M, Khan H, Gul K, Ahmad W. Catalytic efficiency of some novel nanostructured heterogeneous solid catalysts in pyrolysis of HDPE. Polym Degrad Stab. 2013 Dec;98(12):2512-9. doi: 10.1016/j.polymdegradstab.2013.09.009

54. González YS, Costa C, Márquez MC, Ramos P. Thermal and catalytic degradation of polyethylene wastes in the presence of silica gel, 5A molecular sieve and activated carbon. J Hazard Mater. 2011 Mar;187(1-3):101-12. doi: 10.1016/j.jhazmat.2010.12.121

55. Obeid F, Zeaiter J, Al-Muhtaseb AH, Bouhadir K. Thermo-catalytic pyrolysis of waste polyethylene bottles in a packed bed reactor with different bed materials and catalysts. Energy Convers Manag. 2014 Sep;85:1-6. doi: 10.1016/j.enconman.2014.05.075

56. Coelho A, Costa L, Marques MM, Fonseca IM, Lemos MANDA, Lemos F. The effect of ZSM-5 zeolite acidity on the catalytic degradation of high-density polyethylene using simultaneous DSC/TG analysis. Appl Catal A Gen. 2012 Jan;413-414:183-91. doi: 10.1016/j.apcata.2011.11.010

57. Marcilla A, Gómez A, Reyes-Labarta JA, Giner A. Catalytic pyrolysis of polypropylene using MCM41: Kinetic model. Polym Degrad Stab. 2003 Mar;80(2):233-20. doi: 10.1016/S0141-3910(02)004032 
58. Saha B, Ghoshal AK. Model-free kinetics analysis of decomposition of polypropylene over Al-MCM41. Thermochim Acta. 2007 Aug;460(1-2):77-84. doi: 10.1016/j.tca.2007.05.016

59. Obalı Z, Sezgi NA, Doğu T. Catalytic degradation of polypropylene over alumina loaded mesoporous catalysts. Chem Eng J. 2012 Oct;207-208:421-5. doi: 10.1016/j.cej.2012.06.146

60. Sakata Y, Uddin MA, Muto A. Degradation of polyethylene and polypropylene into fuel oil by using solid acid and non-acid catalysts. J Anal Appl Pyrolysis. 1999 Jul;51(1):135-55. doi: 10.1016/S01652370(99)00013-3

61. Caldeira VPS, Santos AGD, Oliveira DS, Lima RB, Souza LD, Pergher SBC. Polyethylene catalytic cracking by thermogravimetric analysis: Effects of zeolitic properties and homogenization process. J Therm Anal Calorim. 2017 Jul;130(3):1939-51. doi: 10.1007/s10973-017-6551-6

62. Edreis EMA, Yao H. Kinetic thermal behaviour and evaluation of physical structure of sugar cane bagasse char during non-isothermal steam gasification. J Mater Res Technol. 2016 Oct-Dec;5(4):31726. doi: 10.1016/j.jmrt.2016.03.006

63. Zhou H, Long YQ, Meng AH, Li QH, Zhang YG. Thermogravimetric characteristics of typical municipal solid waste fractions during co-pyrolysis. Waste Manag. 2015 Apr;38(1):194-200. doi: 10.1016/j.wasman.2014.09.027

64. Kumar S, Panda AK, Singh RK. A review on tertiary recycling of high-density polyethylene to fuel. Resour Conserv Recycl. 2011 Sep;55(11):893-910. doi: 10.1016/j.resconrec.2011.05.005

65. Bai F, Guo W, Lü X, Liu Y, Guo M, Li Q, et al. Kinetic study on the pyrolysis behavior of Huadian oil shale via non-isothermal thermogravimetric data. Fuel. 2015 Apr;146:111-8. doi: 10.1016/j.fuel.2014.12.073

66. Arkhangel'skii IV., Dunaev AV., Makarenko IV., Tikhonov NA, Belyaev SS, Tarasov AV. NonIsothermal Kinetic Methods. Germany: Edition Op. Berlim; 2013.

67. Khawam A, Flanagan DR. Solid-state kinetic models: Basics and mathematical fundamentals. J Phys Chem B. 2006 Jun;110(35):17315-28. doi: 10.1021/jp062746a

68. Bianchi O, Martins JDN, Fiorio R, Oliveira RVB, Canto LB. Changes in activation energy and kinetic mechanism during EVA crosslinking. Polymer Testing, 2011 Sep;30(6):616-24. doi: 10.1016/j.polymertesting.2011.05.001

69. Wang H, Yang J, Long S, Wang X, Yang Z, Li G. Studies on the thermal degradation of poly (phenylene sulfide sulfone). Polym Degrad Stab. 2004 Feb; 83(2):229-35. doi: 10.1016/S01413910(03)00266-0

70. Tiptipakorn S, Damrongsakkul S, Ando S, Hemvichian K, Rimdusit S. Thermal degradation behaviors of polybenzoxazine and silicon-containing polyimide blends. Polym Degrad Stab. 2007 Jul;92(7):1265-78. doi: 10.1016/j.polymdegradstab.2007.03.021

71. Budrugeac P. Some methodological problems concerning the kinetic analysis of non-isothermal data for thermal and thermo-oxidative degradation of polymers and polymeric materials. Polym Degrad Stab. 2005 Mar;89(2):265-73. doi: 10.1016/j.polymdegradstab.2004.09.014

72. Araujo AS, Fernandes VJ, Fernandes GJT. Thermogravimetric kinetics of polyethelyne degredation over silicoaluminophosphate. Thermochim Acta. 2002 Sep;392-393:55-61. doi: 10.1016/S00406031(02)00072-2

73. Erceg M, Kovačić T, Klarić I. Dynamic thermogravimetric degradation of poly(3hydroxybutyrate)/aliphatic- aromatic copolyester blends. Polym Degrad Stab. 2005 Oct;90(1):86-94. doi: 10.1016/j.polymdegradstab.2005.02.014

74. Ozawa T. Thermal analysis — review and prospect. Thermochim Acta. 2000 Jul;355(1-2):35-42. doi: 10.1016/S0040-6031(00)00435-4 